City University of New York (CUNY)

CUNY Academic Works

2017

\title{
Optimal Microgrids Placement in Electric Distribution Systems Using Complex Network Framework
}

Mahmoud Saleh

CUNY City College

Yusef Esa

CUNY City College

Nwabueze Onuorah

CUNY City College

Ahmed Mohamed

CUNY City College

\section{How does access to this work benefit you? Let us know!}

More information about this work at: https://academicworks.cuny.edu/cc_pubs/638

Discover additional works at: https://academicworks.cuny.edu

This work is made publicly available by the City University of New York (CUNY).

Contact: AcademicWorks@cuny.edu 


\title{
Optimal Microgrids Placement in Electric Distribution Systems Using Complex Network Framework
}

\author{
Mahmoud Saleh, Graduate Student Member, IEEE, Yusef Esa, Member, IEEE, Nwabueze Onuorah, \\ Student Member, IEEE, Ahmed A. Mohamed, Senior Member, IEEE
}

\begin{abstract}
This paper provides a new approach to find the optimal location for Microgrids (MGs) in electric distribution systems using complex network analysis. An optimal location in this paper refers to a location that would result in increased grid resilience, reduced power losses, less line loading, higher voltage stability and secured supply to critical loads during power outage. The criteria used to find the optimal placement of MGs were based on the centrality analysis adopted from complex network theory, the center of mass concept used in physics, and the controlled delivery grid (CDG) concept. An IEEE 30-bus system was used as a case study. Results using MATLAB and PowerWorld show the effectiveness of the proposed methodology to be used for MG placement.
\end{abstract}

Index Terms-Communication based control, complex network theory, controlled delivery grid, electric distribution systems; microgrid placement; resiliency.

\section{INTRODUCTION}

$\mathrm{S}_{\mathrm{v} e \mathrm{~s}}^{\mathrm{C}}$ CIENTISTS in a wide variety of fields have, over the Oyears, developed an extensive set of tools: mathematical, computational, and statistical, which aimed at analyzing, modeling, and understanding networks. The study of networks traces its foundations to the development of graph theory, which was first analyzed by Leonhard Euler in 1736 when he wrote the famous Seven Bridges of Königsberg paper [1]. In the context of network theory, complex network is defined to be a graph that is composed from relatively many mutually related nodes (e.g. structural or functional relation) [2], [3]. It is a network with non-trivial topological features that do not occur in simple networks such as random graphs but often occurs in graph modelling of real systems [4]. Based on the prior definitions electric power systems can be categorized as complex networks and analyzed through the lens of network theory [5]-[6].

Electric power system is among the most critical infrastructures. It is undergoing tremendous changes driven by the increase in electricity demand [7], and the worldwide efforts to reduce carbon dioxide $\left(\mathrm{CO}_{2}\right)$ emissions [8], [9]. Moreover, the recent set of natural disasters (e.g. Hurricane Sandy) partially exposed some of the power system vulnerabilities. Therefore, a national call for increasing the grid resiliency, and self-healing capability was raised [10]. This in turn led to the imperative to smart grid, with a major role expected to be played by microgrids. In order to exploit the maximum benefits of microgrids within the electric power system, microgrid location is an important aspect to be investigated.

Previous research focused on finding the optimal location of distributed generation within a microgrid using Metaheuristic methods to minimize the system losses [11]. Some of the preformed research in the literature used complex network analogy with electric power system to conduct transient stability assessment [5], [6]. We used Controlled Delivery Grid (CDG) concept that has been developed in [12], [13] to increase the resiliency within an electric distribution system with high penetration of microgrids [14]. The CDG concept proposes full real-time monitoring and control of the loads by a central controller that processes "requests" from all loads and yield back "grants" according to an energy management algorithm. In this paper, a complex network framework has been established for a modified IEEE 30-bus system, along with the center of mass concept, to determine optimal locations to deploy microgrids. The real-time energy management based on the CDG concept, presented in [14], was used. This paper is aimed to analyze and find the optimal location of microgrids within electric distribution systems.

\section{SYSTEM UNDERSTUDY}

The system understudy is shown in Fig. 1. It represents the IEEE 30-bus standard Test Case. The bus and line data have been extracted from [15]. The system has been modified to represent a blackout condition, by disconnecting the main infeed coming from the grid. The system is divided into three load areas, namely Area 1, Area 2 and Area 3, each with different load profile, as shown in Fig 1. Each bus has a local controller. These controllers, in the case of load buses, send load requests to the central controller. In the case of microgrid buses, these local controllers represent the Microgrid Central Controllers (MGCCs), and send generation requests.

The central controller runs the energy management algorithm in [14]. The algorithm searches for a solution that satisfies all the constraints (i.e. permissible voltage limits, line loading and least losses) with all requested loads granted. If no possible solution exists, the algorithm has to search for a solution with some requests not being fully granted. We assume that each bus controller will send a load request, in the form of a set that contains four load levels with a reasonable difference (e.g. $\geq 10 \%$ ). The grant decision will be based on a priority list in [14], such that Area 1 has the highest priority and Area 3 has the least. In other words, the central controller will attempt to grant all the requested loads. If that is not possible, it will repeat the 


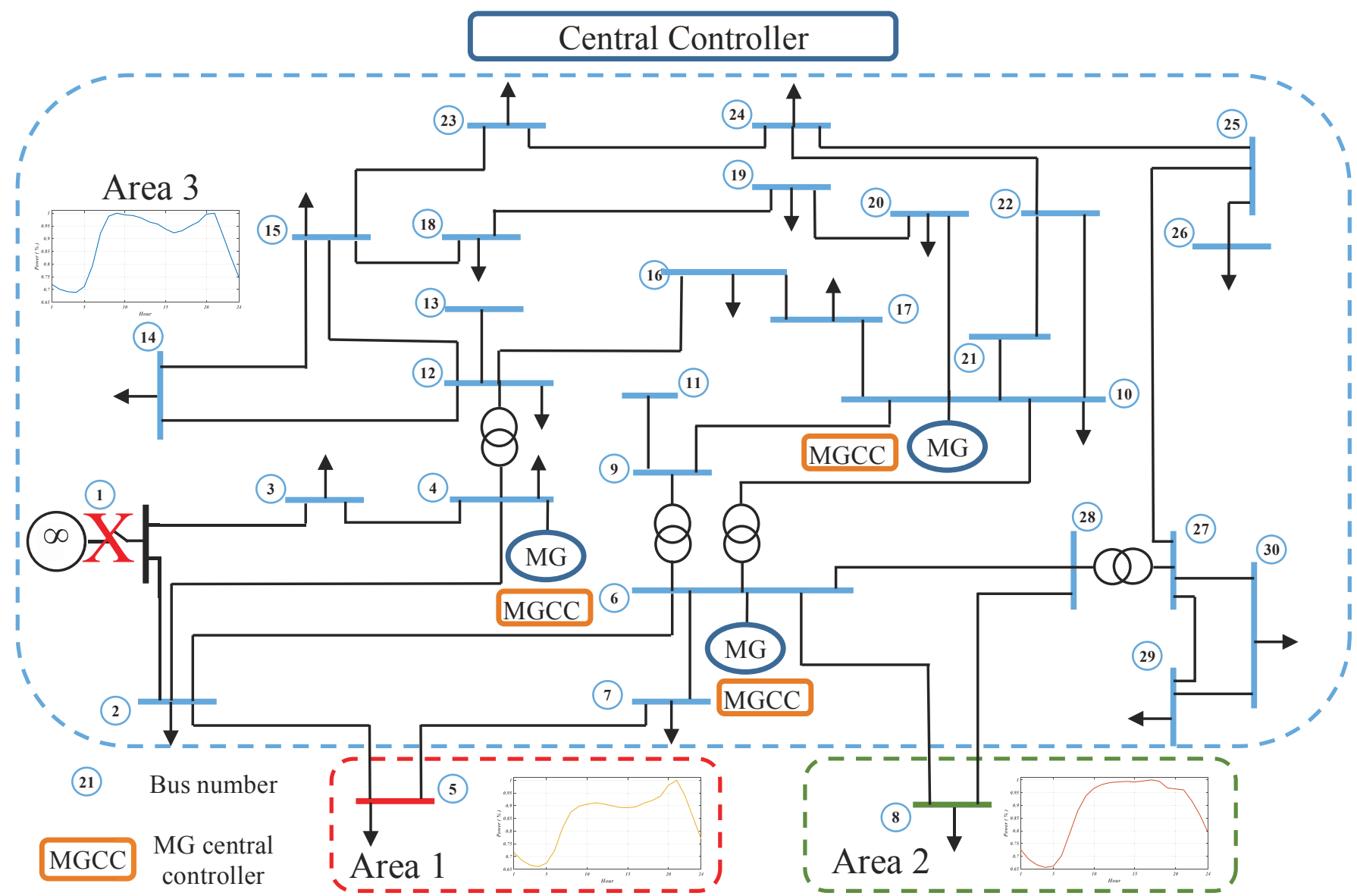

Fig. 1. Modified IEEE 30-bus system.

algorithm for a reduced load, based on their priorities.

In this paper, the location of the microgrids that will enhance the central controller performance will be further examined using complex network framework, which will be described in the following section.

\section{COMPLEX NeTwORK FrAMEWORK}

In order to scrutinize the power system within the context of complex network theory, the first step is to model the system as a graph (i.e. buses as vertices and transmission lines as edges). Fig. 2. shows the corresponding mapped graph from the original IEEE 30-bus system. It contains 30 nodes/vertices and the transmission lines are represented by 41 links/edges, which connect the various nodes. In this paper, we used the complex network framework in its abstract form (i.e. unweighted and undirected) to have some indications about the most central buses in terms of connectivity. In addition, we added different weights to the network edges to explore the effect of the electric system parameters (i.e. normalized impendences and capacities of the lines) on the nodes centrality.

Three main factors have been selected to better understand the centrality in the IEEE 30-bus: betweenness centrality; clustering coefficient; and closeness centrality.

Betweenness centrality is equal to the number of shortest paths from all vertices (i.e. buses) to all others that pass through that vertex. It was devised as a general measure of centrality [16] (i.e. a node with higher betweenness centrality would have more impact over the network). The weight for the edges in betweenness centrality was set to be the normalized admittance of the lines to be more related to the flow of power in the network. For example, the higher the admittance is of the lines connected to a node, the higher the chance that the power flows through this node. Betweenness centrality was calculated using (1).

$c(v)=\sum_{s, t \neq u} \frac{n_{s t}(v)}{N_{s t}}$

Where $n_{s t}(v)$ is the number of shortest paths from $s$ to $t$ that pass-through node $v$, and $N_{s t}$ is the total number of shortest paths from $s$ to $t$.

Closeness centrality is the average length of the shortest paths between the node and all other nodes in the graph [17]. As closeness centrality of a node increases, it indicates how closer it is to the rest of the nodes. The shortest electric path between any two buses is the path, which has the minimum electric distance between them. Therefore, the weight of the edges was selected to be the normalized impedance, since closeness centrality is more related to distance. Closeness centrality was computed using (2).

$c(i)=\left(\frac{v_{i}}{N-1}\right)^{2} \frac{1}{C_{i}}$

Where $v_{i}$ is the number of reachable nodes from node $i$ (not counting $i$ ), $N$ is the number of nodes in graph $G$, and $C_{i}$ is the sum of distances from node $i$ to all reachable nodes. If no nodes are reachable from node $\mathrm{i}$, then $c(i)$ is zero.

Clustering coefficient is a measure of the degree to which nodes in a graph tend to cluster together. The local clustering coefficient of a vertex quantifies the average connection of its neighbor vertices. The overall clustering coefficient of the network and the local clustering coefficient for each node can be calculated as in (3) and (4) respectively. 


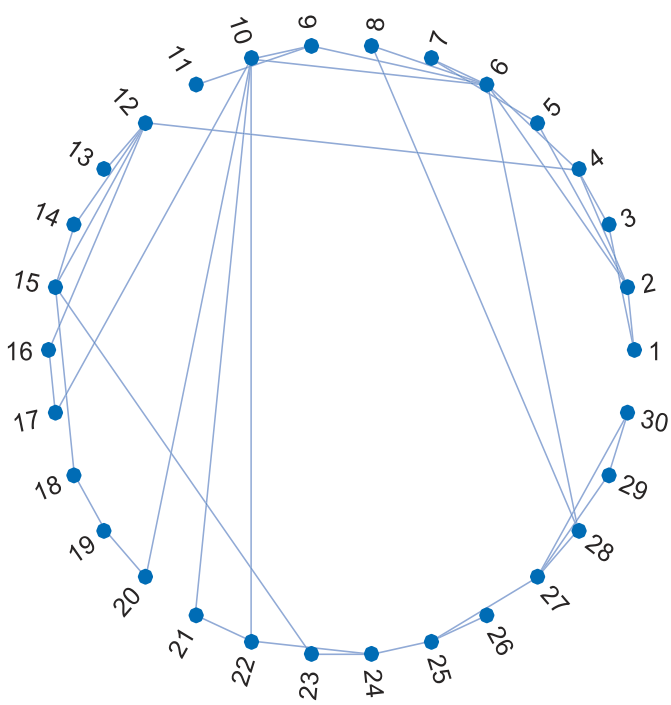

Fig. 2. Mapped graph from the original IEEE 30-bus system.

$C C(v)=\frac{2 N_{v}}{K_{v}\left(K_{v}-1\right)}$

$C C(G)=\frac{1}{n} \sum_{i=1}^{n} C_{i}$

Where $N_{v}$ is the number of links between node $v$ neighbors and $K_{v}$ is the node degree.

The local clustering coefficients was computed to check the clustering tendency of the IEEE 30-bus nodes. Also, from the electric power system point of view, a node has a high clustering coefficient might mean that if it gets disconnected, the power flow can have alternative routes to the surrounding busses. In other words, high LCC of a node can be seen as an indicator it is less central; however, it is not a sufficient indicator by itself. The overall clustering coefficient was calculated to explore the average connectivity of the whole 30 -bus network which is 0.234 . $C C(G)$ equals one (i.e. clique [18]) if all the nodes in the network are connected to each other.

It can be seen from the complex network analysis in Table I that busses 4, 6 and 10 have higher influence than the others. However, if the center of mass analogy is used to locate the center of loads using (6) and (7) in the approximated map from [15], bus 5 will be the closest to the center of the loads. In the following section, we will show how the location of the MGs affects the operation performance.

$x_{l}=\frac{\sum_{i=1}^{n} L_{i} x_{i}}{\sum_{i=1}^{n} L_{i}}$
$y_{l}=\frac{\sum_{i=1}^{n} L_{i} y_{i}}{\sum_{i=1}^{n} L_{i}}$

Where $x_{l}$ and $y_{l}$ are the $x$ and $y$ coordinates of the center of loads, $L_{i}$ is the load connected to bus $i$, and $n$ is the number of buses.

\section{Results And Discussion}

The locations of the MGs determined by the complex network analysis and the center of mass concept (graphically placed near the highest loaded buses) have been tested using the modified IEEE 30-bus described in section II. The system is experiencing a power outage (i.e. main infeed from bus 1 is disconnected). Three different locations for the microgrids were selected to show their impact on the CDG with the algorithm in [14].

Center of mass analogy showed that the center of loads is close to bus 5, therefore, buses 5, 3 and 10 were selected to be the set of locations (L1) to deploy the three microgrids. Buses 3 and 10 were selected with 5 to guarantee that the microgrids are spread over the network. Set of locations (L2) were selected to be buses 27, 29 and 30, which have a high clustering coefficient according to the complex network analysis.. Set of locations (L3) were selected to be buses 4,6 , and 10 which have the highest centrality in the network as described in section III.

Fig. 3 depicts the impact of placing the microgirds at L1. Figs. $3 \mathrm{a}, 3 \mathrm{~b}$ and $3 \mathrm{c}$ show the normalized requested load, and granted power for 24 hours at Areas 1, 2 and 3, respectively. The number of the bus chosen by the algorithm to be the slack bus is shown at each hour. Similarly, Figs. 4 and 5 demonstrate the effect of placing the microgrids at L2 and L3, respectively.

In comparison between Figs. 3, 4 and 5, it shows that L3, which is suggested by the complex network, has the highest grant/request percentage in the three areas (i.e. more loads were supplied during the blackout). In addition, results in Fig. 4 show that high clustering coefficient does not indicate optimal placement. Even during early hours when the loads are low, the central controller was unable to supply the loads in Areas 2 and 3 as shown in Figs. 4b and 4c. By examining results in Fig. 3, it can be seen that L1 is near- optimal location to deploy microgrids. Even though one might think intuitively, placement of microgrids near the highest load should decrease the losses and increase the grant/request percentage, but the network structure may play a significant role. It should be mentioned also that if bus 4 is disconnected (using the PowerWorld model from [15]), a blackout occurs. Similarly, bus 6 causes a blackout. On the other hand, when bus 10 is disconnected, most of the lines in the network were overloaded.

It is worth mentioning that the possible solutions to place an M-number of microgrids in an N-bus distribution system are $\mathrm{M}^{\mathrm{N}}$. Then, in the case of 3 microgrids in 30-bus system, the possible solutions are $3^{30}$, not mentioning the processing time to obtain each solution, which requires tremendous time to find the optimal solution. Using the proposed complex network approach will reduce the possible microgrid placement options, hence, it will reduce the processing time required to find the optimal microgrids location.

\section{CONClusions}

This paper presented a new approach to scrutinize electric distribution systems through complex network framework to obtain indicators about the plausible optimal locations to implement microgrids. The analysis was performed using centrality analysis adopted from complex network theory, the center of mass concept, and the controlled delivery grid (CDG) concept. The IEEE 30-bus Test Case has been used to examine the validity and applicability of the proposed methodology. It should be mentioned that exploring all the possible solutions to place an M-number of microgrids in an $\mathrm{N}$-bus distribution system requires huge amount of time. Complex network theory show substantial contribution regarding the indication of optimal placement of microgrids in a distribution system to increase its resiliency. 


\section{REFERENCES}

[1] "Theory, Culture \& Society 29 (4-5), Special Issue on Topologies of Culture.". Available online at: www.scribd.com/document/125138070/Theory-Culture-Society-29-45-Special-Issue-on-Topologies-of-Culture..

[2] Burian, Jan. Complex Systems Tutorial, Available online at: eldar.cz/cognition/complex/\#Intuitive\%20Definitions\%20of\%20Com plexity.Steen, Maarten van. Graph Theory and Complex Networks: an Introduction. Maarten Van Steen, 2010.

[3] J. Kim, T. Wilhelm, "What is a complex graph?," Physica A, 387 (11) (2008), pp. 2637-2652

[4] ] A. B. M. Nasiruzzaman and H. R. Pota, "Critical node identification of smart power system using complex network framework based centrality approach," in North American Power Symposium (NAPS), 2011, Aug. 2011, pp. 1-6.

[5] ] A. B. M. Nasiruzzaman, and H. R. Pota, "Transient stability assessment of smart power system using complex networks framework," accepted for presentation in IEEE PES GM, Detroit, Michigan, USA, July 24-29, 2011.

[6] "Why smart grids?," 2016. [Online]. Available: http://new.abb.com/smartgrids/why-smart-grids.

[7] J. Gale, "IEAGHG Information paper: 2015-IP29; Emissions Performance Standards," in IEAGHG, 2015. [Online]. Available: http://www.ieaghg.org/docs/General_Docs/Publications/Information Papers/2015-IP29.pdf.

[8] "Fact sheet: President Obama to announce historic carbon pollution standards for power plants," in The White House, whitehouse.gov, 2015. [Online]. Available: https://www.whitehouse.gov/the-pressoffice/2015/08/03/fact-sheet-president-obama-announce-historiccarbon-pollution-standards.
[9] Department of Energy. "Smart Grid." [Online]. Available: https://energy.gov/oe/services/technology-development/smart-grid.

[10] Department of Energy. "Smart Grid." [Online]. Available: https://energy.gov/oe/services/technology-development/smart-grid.

[11] M.H. Moradi, M. Adedini, "A Novel method for optimal DG units capacity and location in micro grids," Int $J$ Electr Power Energy Syst, pp. 236-244, Feb. 2016

[12] R. Rojas-Cessa, Y. Xu and H. Grebel. "Management of a smart grid with controlled-delivery of discrete levels of energy." In IEEE Electrical Power and Energy Conference, 2013 IEEE International Conference on. IEEE, 2013, pp.1-5.

[13] R. Rojas-Cessa, S. Vinit, M. Eugene, B. Divya, K. Justin and H. Grebel. "Testbed evaluations of a controlled-delivery power grid." In Smart Grid Communications (SmartGridComm), 2014 IEEE International Conference on, IEEE, 2014, pp. 206-211.

[14] M. Saleh, Y. Esa, and A. Moahmed, "Energy Management Algorithm for Resilient Controlled Delivery Grids," accepted and to be presented at Industry and Application Society (IAS) conference, Cincinnati, OH, 1-5 October 2017.

[15] Available online at: https://www2.ee.washington.edu/research/pstca/pf30/pg_tca30bus.ht m. Last accessed: 04/06/2017.

[16] F, Linton, "A set of measures of centrality based on betweenness". Sociometry. 40: 35-41, 1977. doi:10.2307/3033543.

[17] G. Sabidussi , "Introduction," in The centrality index of a graph. Psychometrika, 1966.

[18] S, Neil, "Cliques," in Encyclopedia of educational psychology. Sage Publications, 2008

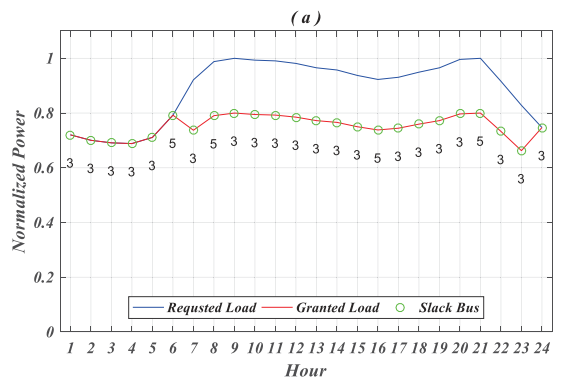

Hour

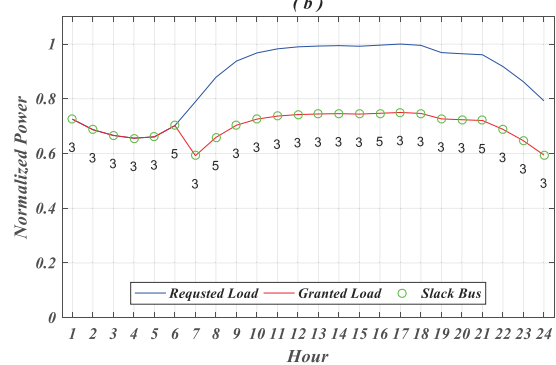

(c)

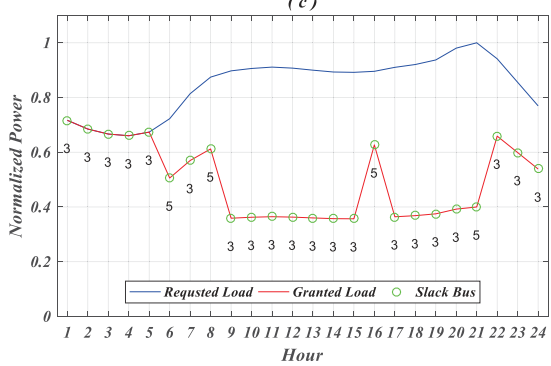

Fig. 3. Normalized requested load, and granted power for 24 hours when MGs placed at L1 for: a) Area 1, b) Area 2 and c) Area 3.
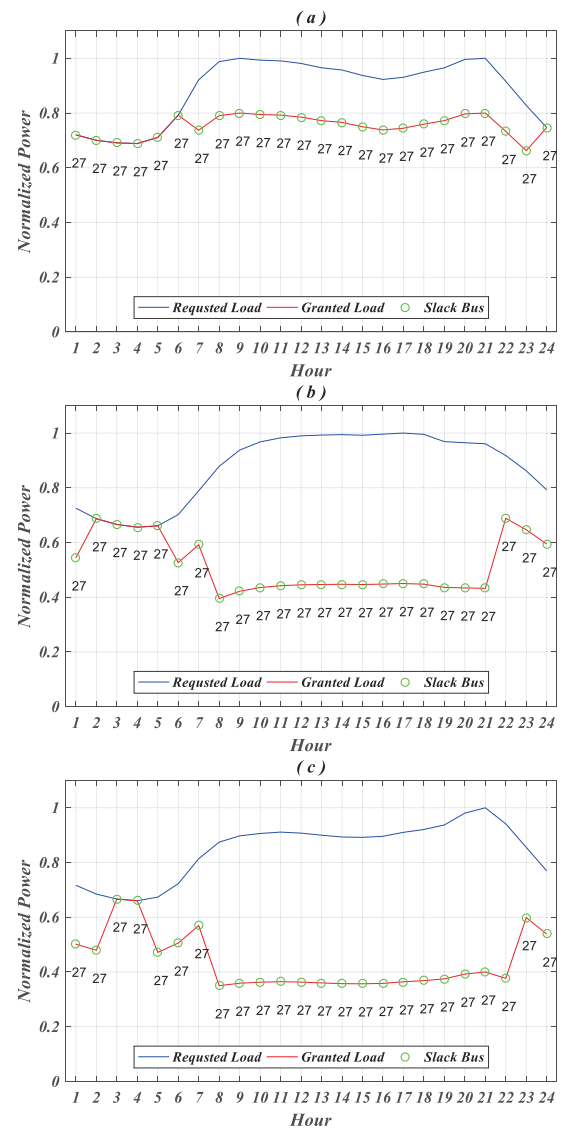

Fig. 4. Normalized requested load, and granted power for 24 hours when MGs placed at L2 for: a) Area 1, b) Area 2 and c) Area 3.

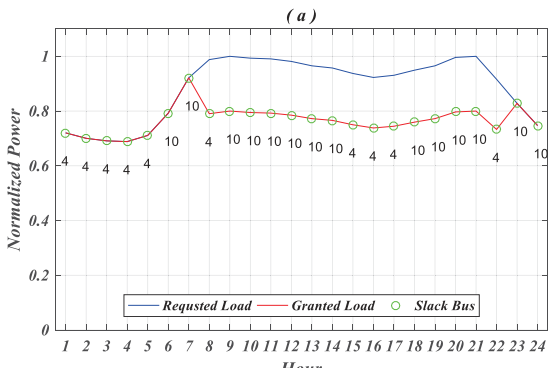

Hour
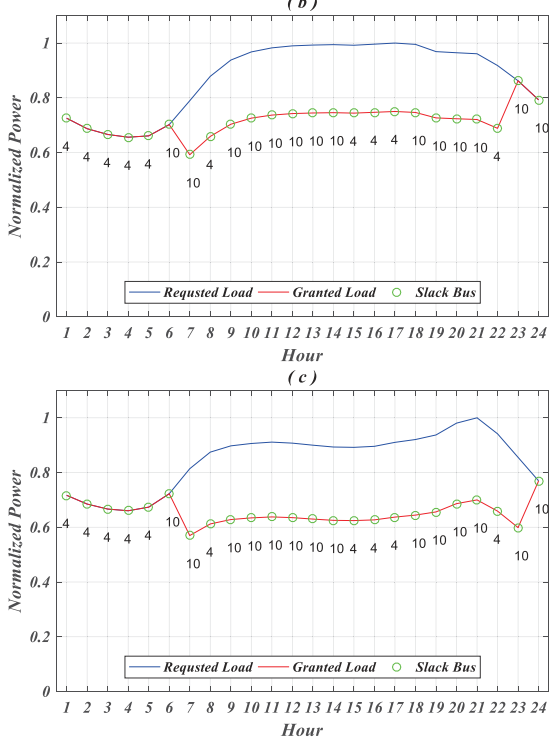

Fig. 5. Normalized requested load, and granted power for 24 hours when MGs placed at L3 for: a) Area 1, b) Area 2 and c) Area 3. 
Table I

Results From Centrality Analysis Using Complex Network Framework

\begin{tabular}{|c|c|c|c|c|c|}
\hline Bus No. & Closeness Centrality & $\begin{array}{c}\text { Average } \\
\text { Closeness } \\
\text { Centrality }\end{array}$ & Betweeness Centrality & $\begin{array}{c}\text { Average } \\
\text { Betweeness } \\
\text { Centrality }\end{array}$ & $\begin{array}{l}\text { Clustering } \\
\text { Coefficinet }\end{array}$ \\
\hline
\end{tabular}
Bus No.

Weight

\begin{tabular}{|c|c|c|c|c|c|c|c|}
\hline 1 & 0.03757 & 0.009346 & 0.023458 & 2 & 1 & 1.5 & 0 \\
\hline 2 & 0.04114 & 0.012346 & 0.026743 & 28 & 40.5 & 34.25 & 0.166667 \\
\hline 3 & 0.037896 & 0.009901 & 0.023898 & 0 & 4 & 2 & 0 \\
\hline 4 & 0.051342 & 0.013333 & 0.032338 & 126 & 89.75 & 107.875 & 0.166667 \\
\hline 5 & 0.04297 & 0.009346 & 0.026158 & 34 & 1 & 17.5 & 0 \\
\hline 6 & 0.052748 & 0.015152 & 0.03395 & 208 & 176.5833 & 192.2917 & 0.142857 \\
\hline 7 & 0.049101 & 0.01087 & 0.029985 & 57 & 8.5 & 32.75 & 0 \\
\hline 8 & 0.036862 & 0.011364 & 0.024113 & 0 & 0 & 0 & 1 \\
\hline 9 & 0.038094 & 0.012195 & 0.025145 & 28 & 28 & 28 & 0.333333 \\
\hline 10 & 0.047808 & 0.013889 & 0.030848 & 161 & 115.6667 & 138.3333 & 0.133333 \\
\hline 11 & 0.026385 & 0.009091 & 0.017738 & 0 & 0 & 0 & 0 \\
\hline 12 & 0.048605 & 0.012048 & 0.030326 & 125 & 87.5 & 106.25 & 0.1 \\
\hline 13 & 0.032721 & 0.009009 & 0.020865 & 0 & 0 & 0 & 0 \\
\hline 14 & 0.04005 & 0.009804 & 0.024927 & 0 & 0 & 0 & 1 \\
\hline 15 & 0.045568 & 0.011111 & 0.02834 & 81 & 54 & 67.5 & 0.166667 \\
\hline 16 & 0.040794 & 0.010101 & 0.025447 & 8 & 10.41667 & 9.208333 & 0 \\
\hline 17 & 0.041507 & 0.011111 & 0.026309 & 18 & 15.91667 & 16.95833 & 0 \\
\hline 18 & 0.040242 & 0.009174 & 0.024708 & 23 & 11.41667 & 17.20833 & 0 \\
\hline 19 & 0.03232 & 0.009174 & 0.020747 & 4 & 11.41667 & 7.708333 & 0 \\
\hline 20 & 0.035456 & 0.010753 & 0.023104 & 7 & 26.25 & 16.625 & 0 \\
\hline 21 & 0.031265 & 0.010638 & 0.020952 & 0 & 0 & 0 & 1 \\
\hline 22 & 0.037667 & 0.011765 & 0.024716 & 42 & 34.91667 & 38.45833 & 0.333333 \\
\hline 23 & 0.036848 & 0.010309 & 0.023579 & 24 & 31.25 & 27.625 & 0 \\
\hline 24 & 0.030453 & 0.011111 & 0.020782 & 54 & 56.41667 & 55.20833 & 0 \\
\hline 25 & 0.025163 & 0.010101 & 0.017632 & 37 & 48.83333 & 42.91667 & 0 \\
\hline 26 & 0.017861 & 0.007874 & 0.012868 & 0 & 0 & 0 & 0 \\
\hline 27 & 0.024411 & 0.010638 & 0.017525 & 54 & 76.83333 & 65.41667 & 0.166667 \\
\hline 28 & 0.044617 & 0.012346 & 0.028481 & 63 & 72.83333 & 67.91667 & 0.333333 \\
\hline 29 & 0.019412 & 0.008264 & 0.013838 & 0 & 0 & 0 & 1 \\
\hline 30 & 0.020425 & 0.008264 & 0.014345 & 28 & 0 & 14 & 1 \\
\hline
\end{tabular}

